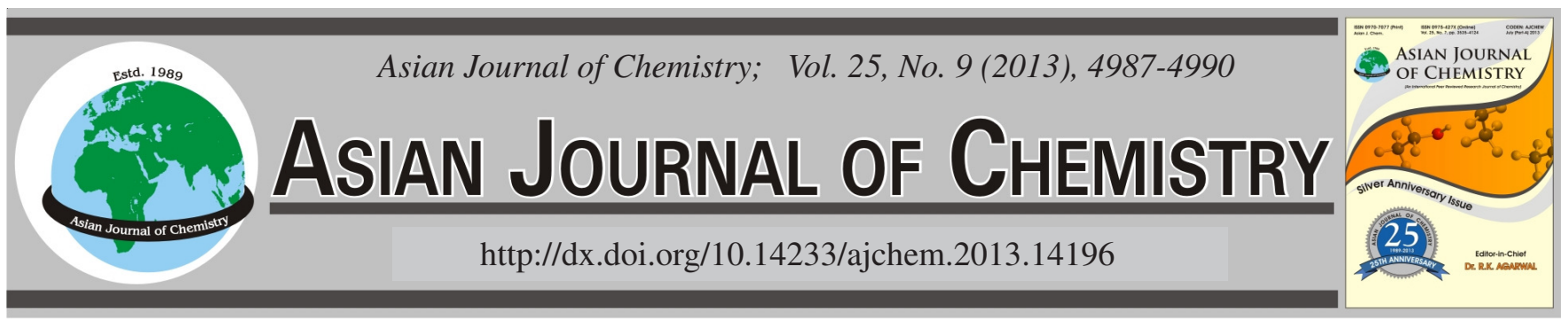

\title{
Influence of Different Organic Waste Materials on Hardening of Micropropagated Tea (Camellia sinensis L.) Clone 'Iran 100'
}

\section{Reza Azadi Gonbad ${ }^{1}$, Uma Rani Sinniah ${ }^{1, *}$, Maheran Abdul Aziz ${ }^{2}$ and Rosfarizan Mohamad ${ }^{3}$}

\author{
${ }^{1}$ Department of Crop Science, Faculty of Agriculture, University Putra Malaysia, 43400 Serdang, Malaysia \\ ${ }^{2}$ Department of Agriculture Technology, Faculty of Agriculture, University Putra Malaysia, 43400 Serdang, Malaysia \\ ${ }^{3}$ Department of Bioprocess Technology, Faculty of Biotechnology and Biomolecular Sciences, University Putra Malaysia, 43400 Serdang, \\ Malaysia
}

*Corresponding author: Fax: +60 3 89474918; Tel.: +60 3 89474839; E-mail: umarani@ agri.upm.edu.my

\begin{abstract}
In order to reduce mortalities of the tea plantlet Clone Iran 100 during the hardening stage after acclimatization from tissue culture, the effect of different organic materials on rooting of acclimatized plantlets was investigated. Organic materials used were urban waste material, farm yard manure, mushroom media and tea waste at the level of $0,25,50$ or $75 \%$ (v/v) per pot mixed with soil. Completely randomized block design with factorial arrangement was used and replicated thrice. During the acclimatization period, dry and fresh weight of the root and shoot, length of the root and shoot, number of leaves and roots along with survival rate were measured and recorded. The final outcome showed that tea waste treatment at the level of $25 \%$ had the highest survival and is considered as the best treatment for hardening of tea.
\end{abstract}

Key Words: Acclimatization, Media, Survival, Tea, Tissue culture.

\section{INTRODUCTION}

Tea is the second most popular non-alcoholic beverage, after water, heavily consumed by majority of the world population. It is processed from the young leaves and leaf buds of Camellia sinensis (L.) O. Kuntze, belonging to the family Theaceae. Based on the type and level of processing, various categories of tea are obtained namely white tea, green tea, black tea, oolong tea ${ }^{1}$. Tea was mainly cultivated and refined in China and Japan before the period of Christ. However, today, tea is also cultivated in Sri Lanka, Malaysia, East Africa, India, South America, Iran and Indonesia, since these regions have appropriate climate for growing this crop. Iran is listed number 14 in relation to tea producing countries ${ }^{2}$ based on land area cultivated with tea. However, in recent years the productivity has shown a decline ${ }^{3}$ mainly attributed to tea gardens maintaining old plants. In general, the economic life of tea plants is about 30 years, beyond which the yield has been reported to deteriorate both in quantity as well as quality. Survey has shown that most of the tea plants in Iran are more than 80 years old, hence, the lower yield has been observed in recent years. Based on research and development, new clones have been discovered, among which tea clone Iran 100 has emerged as a potential clone with high yielding ability as well as with high chemical content ${ }^{3}$. Hence, there is a necessity to find a reliable propagation method of multiplying the clones for commercial establishment of farms $\mathrm{s}^{4}$. For tea propagation, traditional techniques such as budding, layering, semi-mature cuttings and grafting have been utilized with propagation by means of cutting being the most dominant ${ }^{5}$. However, this method is hindered by the low survival of the cuttings as well as the time taken for rooting. Thus, the production of a large number of plants using conventional techniques was rendered as economically unfeasible. To date, large numbers of planting materials are being produced using suitable in vitro propagation method which also eliminates many of the problems related with the hardening and rooting of woody stem cuttings. Often tissue or in vitro culture is done via multiple stages which include sterilization and establishment of culture, multiplication, rooting and finally acclimatization. Various researches have been carried out in relation to establishing protocols for in vitro production and multiplication of tea plantlets ${ }^{6}$. The establishment of such protocol need to be accompanied by good establishment of plants in the nursery prior to transplanting. Severe loss can occur due to undeveloped acclimatized plantlets, as this stage is highly influenced by the use of appropriate medium which can facilitate not only rooting but absorption of nutrient for quick establishment of the plant. The success 
of hardening of the tea plantlets in nursery is usually dependent on media having sandy loam with low humus content. Because of the drainage and low aeration, the usage of clay soils in nurseries is inappropriate ${ }^{7}$. According to Bidarghfard ${ }^{8}$ the tea rooting is dependent on type of media used. Recently, the use of organic material as a component of media for acclimatization has become a subject of interest for tissue culturists. As reported $^{9}$ the presence of organic materials, nutrients and soil physical properties are enhanced through the utilization of waste materials in the media type. The conditions for growing plants can be further improved by increasing unrefined deposits caused by recuperating soil $\mathrm{pH}^{10}$. The intensity of nitrogen, organic anions, ash alkalinity, $\mathrm{pH}$ of soils and cations like $\mathrm{Mg}, \mathrm{Ca}$ and $\mathrm{K}$, result in determining the effect of unrefined material at soil acidity change ${ }^{11-14}$. Although several researches have been conducted in order to determine the rooting of tea cutting ${ }^{8,15-18}$ but to date not much information is available on the use of organic waste material as potting media for hardening of tissue culture produced plantlets. The available report on acclimatization of micropropagated tea clones on soil and organic peat media thus far has recorded a maximum survival of $60 \%$. The purpose of the present research was to further investigate the role played by the various organic matter as well as different ratios in order to increase rooting and to increase survival of micropropagated plantlets.

\section{EXPERIMENTAL}

Shoots of tea clone Iran 100 (Camellia sinensis (L.) O. Kuntze) were initially obtained from the Tea Research Institute, Iran and established in field 2, University Putra Malaysia, Selangor, Malaysia. The nodal segments containing axillary buds from these plants were used as the explant source. Auxillary buds (about $1-1.5 \mathrm{~cm}$ in length) were excized from nodal segments. These were then soaked in Tween 20 for 15 min followed by surface sterilization with $20 \%$ (v/v) clorox solution $(0.53 \%$ sodium hypochlorite) for $15 \mathrm{~min}$. All traces of clorox solution were then washed off with sterile distilled water. Explants were cultured on Murashige and Skoog (1962) (MS) medium supplemented with 6-benzylaminopurine (BAP) at $3 \mathrm{mg} / \mathrm{L}$ in combination with gibberellin $\mathrm{A}_{3}\left(\mathrm{GA}_{3}\right)$ at 0.5 $\mathrm{mg} / \mathrm{L}$ and sucrose (3\%; w/v) and solidified using agar $(0.8 \%$; $\mathrm{w} / \mathrm{v}$ ) for multiplication of shoots. Sub culturing was done at regular intervals of 4 weeks and the induced shoots were transferred to fresh media with the same formulation. Upon obtaining sufficient multiplication, the shoots measuring 2-3 $\mathrm{cm}$ in height were subjected to treatment with indole-3-butyric acid (IBA) solution at $300 \mathrm{mg} / \mathrm{L}$ for $30 \mathrm{~min}$ and then transferred to $1 / 2$ strength Murashige and Skoog medium without growth regulators to initiate rooting.

After 60 days of culture, healthy plantlets with 4-6 leaves and well-developed roots were removed from the media, washed with fungicide and transplanted into polybags $(14 \times$ $16 \mathrm{~cm}$ dimensions) containing organic materials namely urban waste material, farm yard manure, mushroom media or tea waste (Fig. 1) with ratios of $0,25,50$ or $75 \%(\mathrm{v} / \mathrm{v})$ per pot with soil supplementation. In every polybags, only one young plant is planted and three such bags were utilized in each treatment. The length of root and shoot, dry and fresh weight of the root and shoot, the survival rate and the number of leaves and roots were also recorded after 1 year. This experiment was repeated twice. The plants were maintained in the greenhouse under controlled environmrnt [i.e. $25 \pm 2{ }^{\circ} \mathrm{C}$, $16 \mathrm{~h}$ photoperiod $\left(55 \mu \mathrm{mol} \mathrm{m} \mathrm{m}^{-2}\right)$ by cool, white fluorescent lamp and $70 \%$ relative moisture or humidity] and were watered after every two days until it was ready for final transfer to the field.
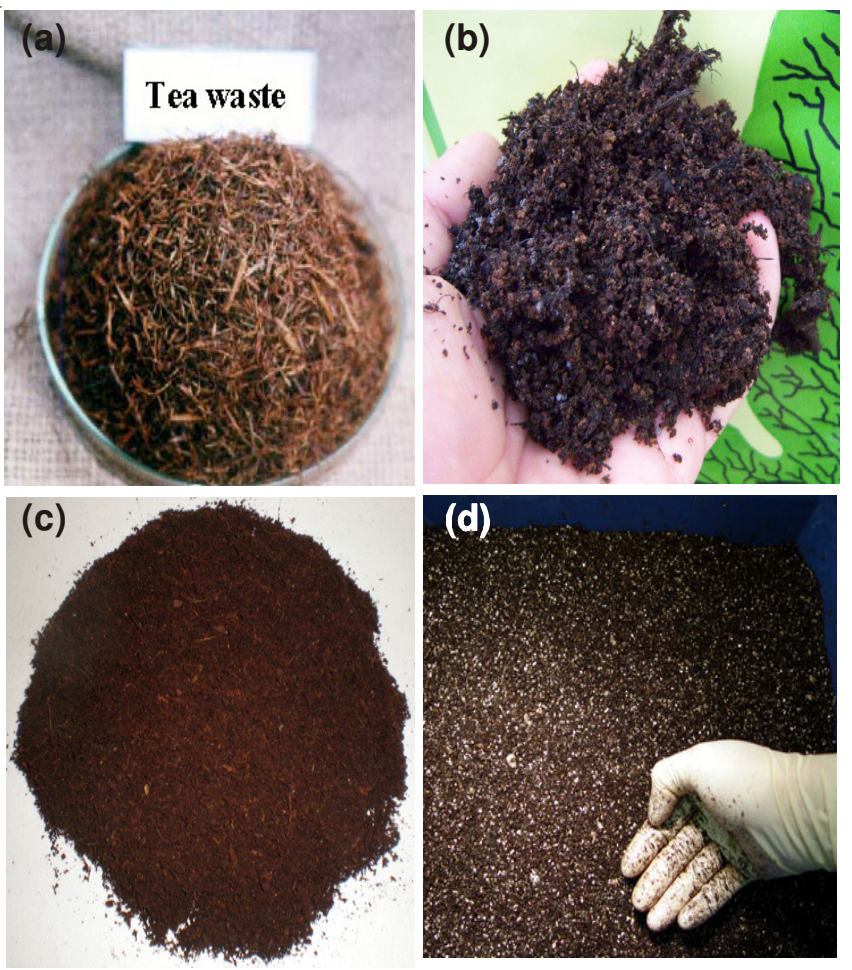

Fig. 1. Organic waste materials (a) tea waste (b) urban waste material (c) farm yard manure and (d) mushroom media

The collected data was analyzed by SAS statistical software and the mean of data was evaluated by the Tukey's Test using the probability of $1 \%$.

\section{RESULTS AND DISCUSSION}

A complete tissue culture protocol often encompasses the rooting as well as acclimatization of the in vitro propagated plantlets. In this study IBA at $300 \mathrm{mg} / \mathrm{L}$ used for root induction of in vitro established tea plantlets showed positive response. Root initiation was observed as early as 1 week after inoculation and further elongation and multiplication was obtained until 2 months (Fig. 2a). Well rooted plantlets are necessary before they can be acclimatized ${ }^{20}$. In this study the use of different organic materials on survival of tea plantlets were tested.

The results of analysis of variance (ANOVA) (Table-1) showed significant difference among various organic materials and their levels on the percentage of survival. However, there was no interaction between various organic materials and their levels on the percentage of survival and number of roots. The highest survival $(72.2 \%)$ was obtained in medium consisting of tea waste followed by $58.3,38.9$ and $33.4 \%$ of survival in 


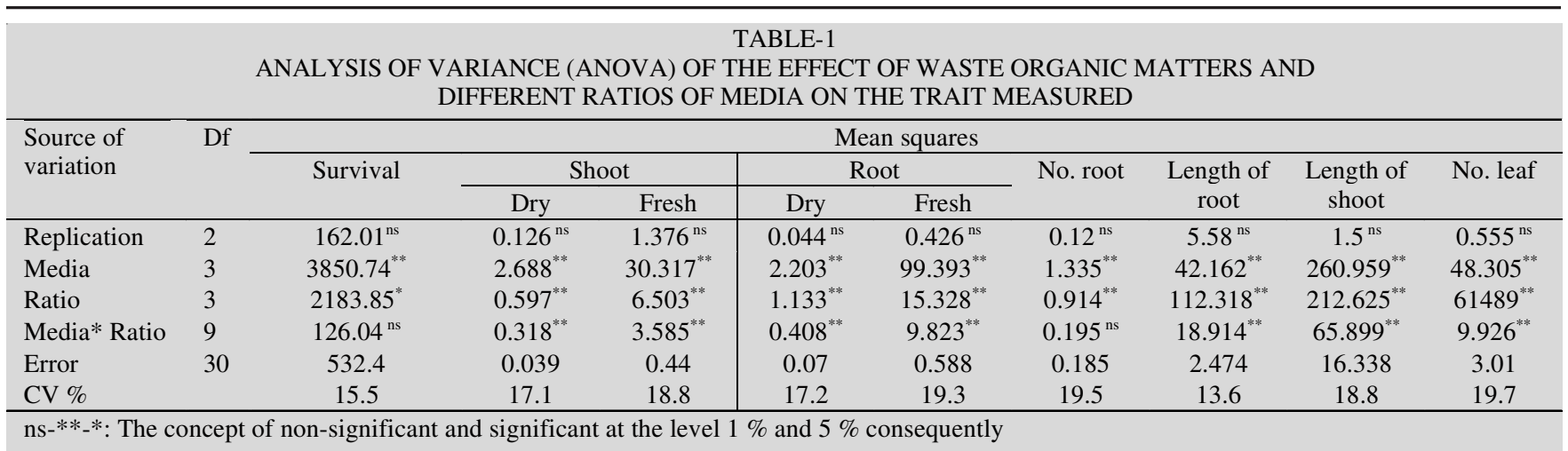

farm yard manure, urban waste and mushroom media, respectively by the end of 6 months (Fig. 2b and Fig. 3). The results indicate that medium containing $25 \%$ organic matter had the best survival (Fig. 4) as well as good plant development based on other morphological parameters considered. The efficiency of tea extract on acclimatization of micropropagated plants was also reported earlier ${ }^{21}$ where the positive influence of tea extract on retention of optimum humidity and induction of new rhizosphere under ex vitro condition was observed.
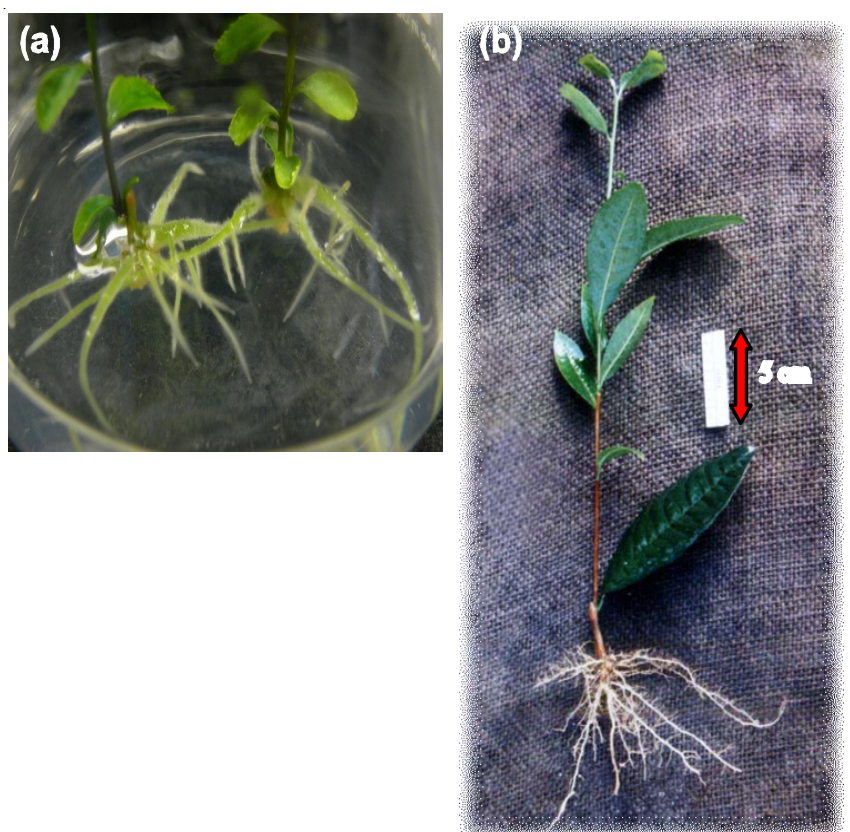

Fig. 2. Comparison of root formation after different times (a) One month in tissue culture medium, (b) Six months after acclimatization

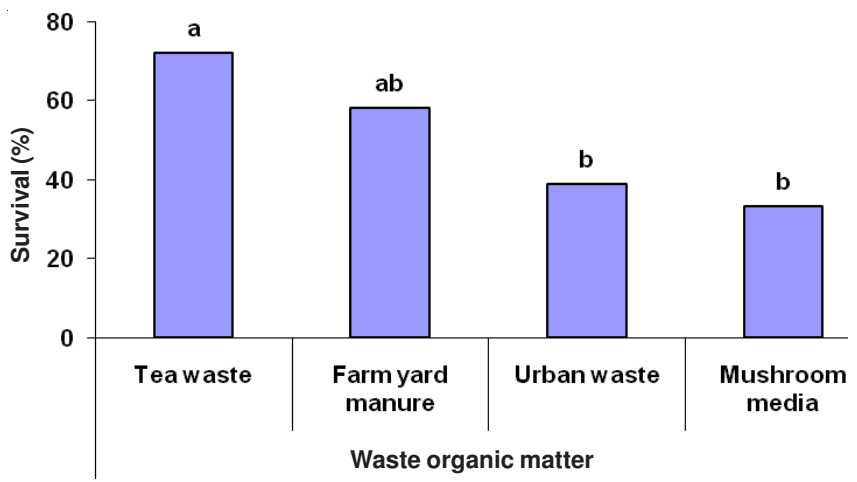

Fig. 3. Comparison of effects of various organic materials on the percentage of survival

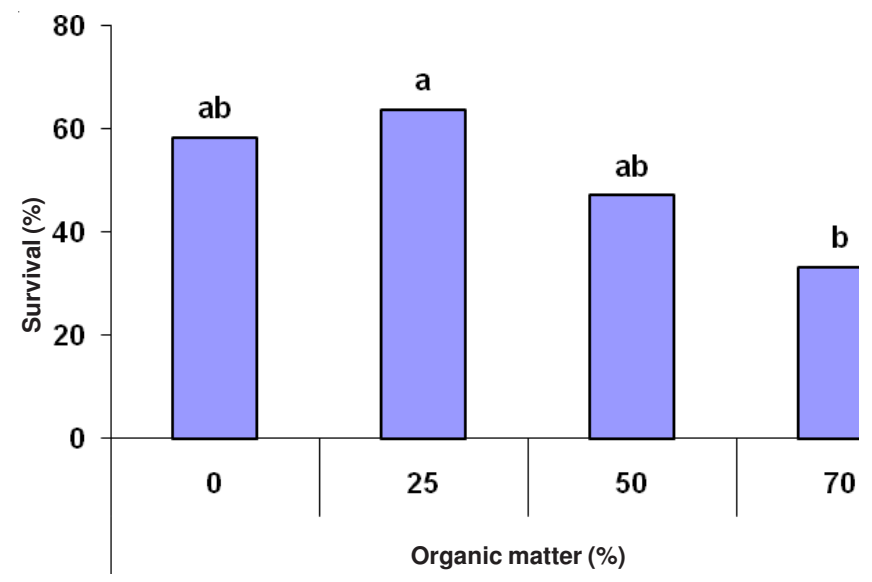

Fig. 4. Comparison of effects of different ratios on the percentage of survival

The correlation of electrical conductivity, growth factors and $\mathrm{pH}$ were computed to determine the relationship among them. The results showed that among these factors with plant growth parameters, there was a significant negative relationship. The results showed that the correlation of $\mathrm{pH}$ with electrical conductivity was significant and positive, but with the other traits were negative and significant. Additionally, with all other characteristics electrical conductivity tends to show a negative correlation (Table-2).

The results presented in Table- 1 shows that the main effects of various organic materials and their ratios and their interaction were significantly different for the remaining morphological parameters. The highest number of leaves was obtained for both $25 \%$ tea waste and farm yard manure compared with other treatments (Fig. 5). It was found that increasing the amount of organic matter from 25 to $75 \%$ resulted in reduction in the number of leaves. Mushroom media and urban waste did not influence the number of leaves, significantly, as compared to the treatments based on farm yard manure and tea waste. In every treatment, the increase from 0 to $25 \%$ in organic matter resulted in improved number of leaves whereas the increase from 25 to $75 \%$ showed a reduction. The farm yard manure composition of $25 \%$ on the length of the root is significantly different as compared to other related treatment (Fig. 6). Raising the rate of farm yard manure to $50 \%$ resulted in decreased length of root, presumably due to the toxicity resulted from higher concentration of farm yard manure.

The ANOVA result (Table-1) and means comparison showed the effect of the organic matter waste at $25 \%$ on most of the parameters was significantly higher in comparison to 
TABLE-2

CORRELATION OF TRAITS HAS BEEN TESTED

\begin{tabular}{ccccccccc}
\hline & Dry Shoot & Fresh Shoot & Dry Root & Fresh Root & No. root & Length of shoot & Length of root & No. leaf \\
\hline $\mathrm{pH}$ & $-0.578^{*}$ & $-0.816^{* *}$ & $-0.838^{* *}$ & $-0.838^{* *}$ & $-0.893^{* *}$ & $-0.691^{* *}$ & $-0.724^{* *}$ & $-0.449^{*}$ \\
$\mathrm{EC}$ & $-0.828^{* *}$ & $-0.561^{*}$ & $-0.603^{*}$ & $-0.603^{*}$ & $-0.514^{*}$ & $-0.591^{*}$ & $-0.602^{*}$ & $-0.601^{*}$ \\
\hline$* * *$ : The concept of significant at the level 1\% and 5\% consequently & & & &
\end{tabular}

the media without organic matter, but, further increase in organic matter waste (to $50 \%$ ) resulted in reduction of performance which resulted in necrosis. The higher organic matter content might reduce the water absorption attributed to the declined performance of plantlets during acclimatization. Hence, the likelihood to increase the elements' solubility along with its high intensity in soil solution can adversely affect plants. The reduction in dry and fresh weight of root seems to be influenced by the high amount of soil acidity. Generally, the promotive effects of different organic matter waste on the development of acclimatized plantlet can be categorized as below:

tea waste $>$ farm yard manure $>$ mushroom media $>$ urban waste materials

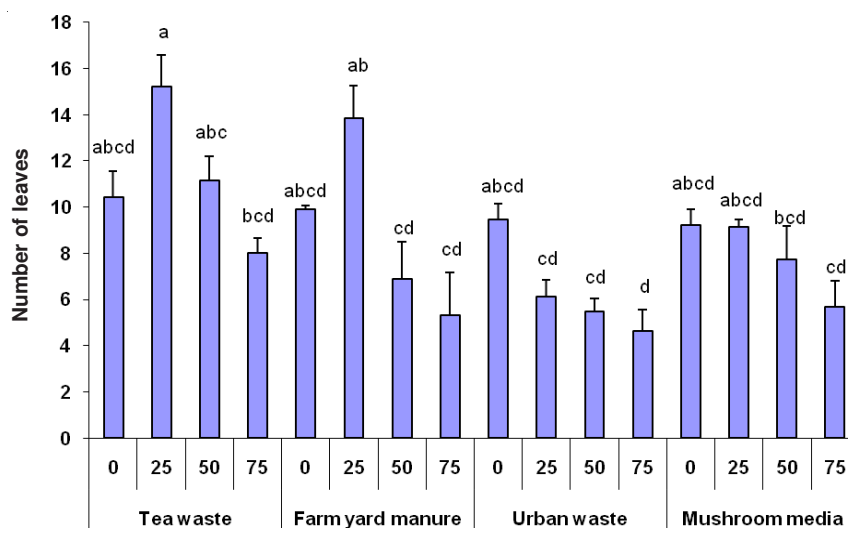

Fig. 5. Comparison of effects of various organic materials and their ratios on number of leaf

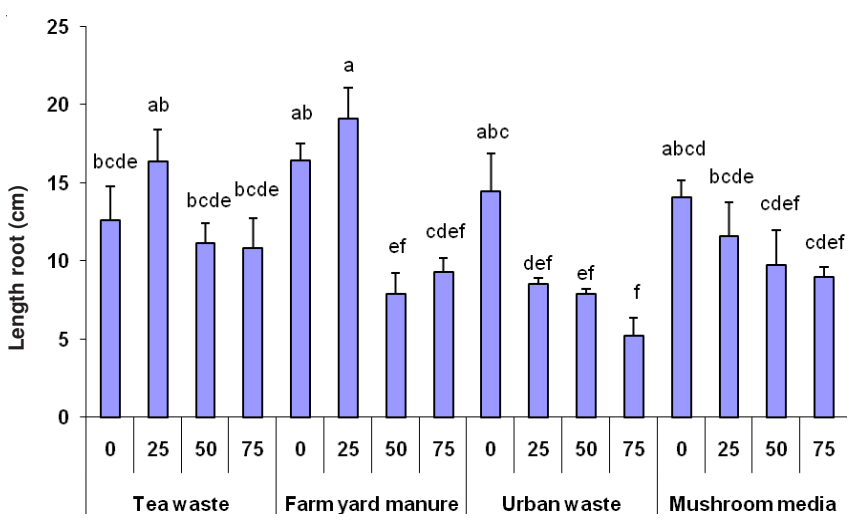

Fig. 6. Comparison of effects of various organic materials and their ratios on length of root
The results also revealed that the effect of adding organic matter higher than $25 \%$ of tea waste, did not show the positive and significant impact on growth and development of plants during acclimatization in comparison to control media (soil without any organic matter).

Out of all the treatments done with the presence of various levels and the types of organic matters, tea waste $25 \%$ proved to be the best treatment for acclimatization. The optimum $\mathrm{pH}$, soil porosity, stimulated soil microbial populations, lower electrical conductivity and enhanced water holding capability might influence the favourable result during acclimatization in tea waste media. However, all morphological traits affected by farm yard manure were similar to that of tea waste, except that due to higher $\mathrm{pH}$ of the former the seedling morphology was compromised. Therefore, it is recommended that for acclimatization and hardening of tea plants the soil media is amended with tea waste at the ratio of 1:3 (1 tea waste: 3 soil) for increased rooting and plant growth. In addition this will also provide a means to reuse tea waste materials.

\section{REFERENCES}

1. K. Willson, Coffee, Cocoa and Tea, (CAB International, 1999).

2. F. Ansari, R. Norbaksh and K. Daneshmandirani, Iran. J. Environ. Health Sci. Eng., 4, 243 (2007).

3. R.A. Gonbad and A.F. Chokami, Asian J. Chem., 21, 3298 (2009).

4. Y. Nakamura, Jpn. Agric. Res. Q, 25, 185 (1991).

5. H.T. Hartmann, D.E. Kester, F.E. Davies and R. Geneve, Plant Propagation: Principles and Practices, (Prentice-Hall, New Jersey, 2002).

6. T.K. Mondal, A. Bhattacharya, M. Laxmikumaran and P.A. Singh, Plant Cell, Tissue and Organ Culture, 76, 195 (2004).

7. O.M. Prakash, P.K. Nagar and P.S. Ahuja, J. Plantation Crops, 29, 56 (2001).

8. S. Bidarighfard, Islamic Azad University (1997).

9. C. Weeraratna, M. Watson and D. Wettasingha, Tea Quarterly, 50, 131 (1981).

10. R. Kretzschmar, H. Hafner, A. Bationo and H. Marschner, Plant Soil, 136, 215 (1991).

11. A. Noble, I. Zenneck and P. Randall, Plant Soil, 179, 293 (1996).

12. S. Pocknee and M.E. Sumner, Soil Sci. Soc. Am. J., 61, 86 (1997).

13. C. Tang and Q. Yu, Plant Soil, 215, 29 (1999).

14. F. Yan and S. Schubert, Plant Soil, 220, 279 (2000).

15. J.E.E.S. Chen, F.M. Thseng and W. Ko, Hort. Sci., 25, 305 (1990).

16. E. Altinda and F. Balta, Turk. J. Agric. Forestr., 26, 195 (2002).

17. C.H. Fong, Hort. Abstr., 62-6, 5316 (1992).

18. A. Waheed, H. Ahmad, F. Hamid and N. Ahmad, Sci. Technol. Develop., 29, 15 (2010).

19. T. Jha and S.K. Sen, Plant Cell Reports, 11, 101 (1992).

20. S. Gantait, N. Mandal, S. Bhattacharyya and P.K. Das, Plant Tissue Cult. Biotechnol., 18, 113 (2009).

21. S. Gantait, N. Mandal, S. Bhattacharyya and P.K. Das, In Vitro Cellular Develop. Biol. Plant, 46, 537 (2010). 